\title{
Effect of various chemical treatments of Prosopis juliflora fibers as composite reinforcement: physicochemical, thermal, mechanical, and morphological properties
}

\begin{abstract}
The present environmental regulations enforced by the government authorities have made the investigators around the globe to make use of more and more green materials particularly in composite systems. In this context, natural fibers play an important role and proven to be excellent reinforcements in polymer matrices. However, these natural fibers have got one major limitation: their incompatible hydrophilic behavior which affects their bonding with hydrophobic matrixes. Researchers over the years have come up with several fiber surface modification processes to overcome this defect. So, in this present study, the effect of various chemical treatments like alkaline, benzoyl peroxide, potassium permanganate, and stearic acid on Prosopis juliflora fibers has been discussed. These various chemical treatments on the fiber surfaces impacted on their structure, composition, and properties which were discovered through chemical analysis, Fourier transform-infrared, X-ray diffraction, thermogravimetric analysis, differential scanning calorimetry, scanning electron microscopy, and tensile testing.
\end{abstract}

Keyword: Chemical properties and density analysis; FTIR; XRD; TGA and DSC; SEM 